\title{
48
}

\section{Size Estimation for C Programs with Different Applications}

\author{
Christopher J. Lokan
}

Department of Computer Science, Australian Defence Force Academy, Canberra, Australia.

\begin{abstract}
Cost estimation is an important aspect of the management of software development projects. Most cost estimation models require an estimate of the size of the program to be developed. Accurate size estimation is thus critically important. Earlier work has shown that weighted counts of conceptually unique variables (where each variable is weighted by the number of functions in which it is referenced) are good predictors of the final size of Pascal and C programs. Simple equations using only that information give acceptable accuracy, even across a range of sizes and applications. For Pascal programs, the accuracy is acceptable by the end of high level design; for C programs, it is acceptable by the end of detailed design. In this study, C programs are classified by application, instead of being treated as a single class. Different estimation equations are then used for programs in each class. Overall estimation accuracy improves at each stage of design. A broad classification scheme is sufficient to give estimates of acceptable accuracy by the completion of high level design.
\end{abstract}

Keyword Codes: D.2.8; K.6.1.

Keywords: Software Engineering, Metrics; Project and People Management.

\section{INTRODUCTION}

An important aspect of software project management is the assessment and allocation of the resources required during development. Numerous software cost estimation models exist to help managers with this task. An essential input to all such models is an estimate of the size of the software to be developed. Accurate estimation of software size is thus vital. Unfortunately it is very difficult, especially during the early stages of development.

Many approaches to size estimation are used in industry. Several are supported by commercial size estimation tools and services. Most involve subjective estimation, particularly through comparison with completed projects whose size is known. An alternative approach, in which this work falls, is to develop algorithmic models that predict the size of a program from quantitative measurements of some of its attributes (see for example $[1,2,3,4,5,6,7]$ ).

The research described here concentrates on the variables in a program as the key factor. Lokan and Conte $[8]$ have shown that very simple equations can estimate the final size of Pascal programs with acceptable accuracy, from as early as the completion of high level design, across a range of program applications and sizes. These equations were based on a weighted count of conceptually unique variables, in which each variable in a program was weighted by the number of procedures in which it was used. A similar study of C programs [9] supports these findings, except that accuracy was not quite acceptable after high level design (by detailed design it was acceptable). 
Figure 1: LOC vs. weighted variables
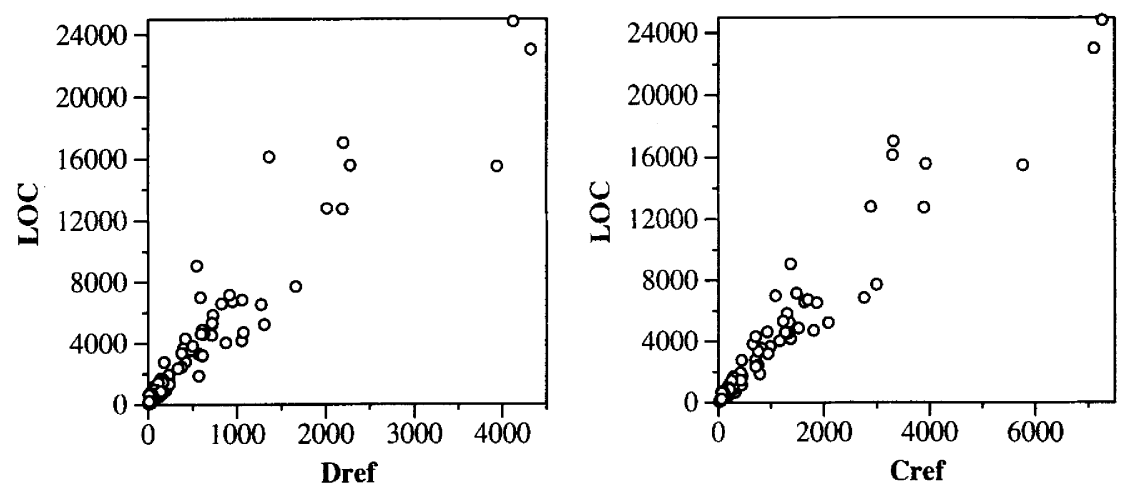

This study looks more closely at the C programs, to try to improve the accuracy of the size estimates by classifying programs into groups for estimation. Rather than considering all programs as one class, with a single estimation equation for them all, separate estimation equations are used for programs in different classes. The programs are classified by application.

\section{METHOD OF STUDY}

\subsection{Data collection}

One hundred and fourteen $\mathrm{C}$ programs, covering a broad range of applications, were analyzed. In total the programs amounted to almost 340,000 lines of source code. They ranged in size from 71 to 24,836 lines, with a mean of 2,975 and a median of 998 lines.

Three attributes were measured for each program: $L O C$ (the number of source lines, excluding blank lines and lines that only contain comments), Dref and Cref (weighted counts of the conceptually unique variables ${ }^{1}$ identifiable after high level design and detailed design respectively). Each identifier was weighted by the number of functions in which it was used. The difference between Dref and Cref is that Cref includes local names within functions, and implementation-dependent constants ( $e g$ array sizes), while Dref does not.

Figure 1 plots the size of each program against Dref and Cref. Both plots show clear linear relationships; the scatter is slightly wider with Dref.

\subsection{Classifying programs by application}

The sample programs were classified into fifteen types of application. These were grouped further into four broad classes: computation-intensive programs, interactive programs with a rich user interface, batch-oriented file translation programs, and system programs. Table 1 summarizes the programs in each class.

\footnotetext{
1 "Variables" includes identifiers defined as variables, struct fields, or values of an enumerated type; identifiers that are passed as parameters in function calls; identifiers defined in \#define directives. System-defined names, like those defined in files such as stdio.h, were not counted. Neither were identifiers declared by the programmer but. not used.
} 
Table 1: Summary of the programs in each classification

\begin{tabular}{llcrrrr}
\hline Class & Application & No. of & \multicolumn{4}{c}{ Program sizes } \\
\cline { 4 - 7 } & & programs & Mean & Median & Smallest & Largest \\
\hline \multirow{3}{*}{ Computation- } & astronomy & 4 & 5073 & 5073 & 3311 & 6835 \\
intensive & cryptography & 5 & 4353 & 5063 & 698 & 6589 \\
& data analysis & 10 & 3597 & 1578 & 305 & 2764 \\
& simulation & 3 & 1775 & 530 & 151 & 23022 \\
& email, news & 5 & 13889 & 16132 & 23.556 & 24836 \\
\hline \multirow{3}{*}{ Interactive: } & communications & 3 & 5500 & 7725 & 4615 & 7161 \\
sophisticated & database & 6 & 1356 & 951 & 683 & 3360 \\
user interface & editor & 7 & 7723 & 6733 & 783 & 15511 \\
& games & 4 & 5471 & 2420 & 1464 & 15578 \\
\hline \multirow{3}{*}{ File or program } & file compression & 7 & 3533 & 1705 & 1156 & 7718 \\
translation & filter & 9 & 836 & 295 & 122 & 3549 \\
& parser & 9 & 1839 & 1085 & 199 & 6995 \\
& translator & 10 & 1219 & 859 & 322 & 4181 \\
\hline System & system & 30 & 1086 & 580 & 71 & 6536 \\
\hline Total & & 114 & 2975 & 998 & 71 & 24836 \\
\hline
\end{tabular}

There are two reasons for grouping programs into broad classes as well as individual applications. First, it is desirable to have estimation models that are as general as possible; broad classifications should be investigated before more detailed ones. Second, each class that is analyzed separately needs to be large enough for the statistics to have some meaning.

\subsection{Estimation equations}

Simple linear equations are desirable, as they are intuitive and easy to apply. Earlier work [8.9] has shown that other forms of equation are no more accurate than linear equations. Hence only linear equations were sought here.

The measures used to evaluate the accuracy of each equation were the mean magnitude of relative error (denoted $\overline{M R E}$ ), and the proportion of programs predicted with $M R E \leq .30$ (denoted Pred( .30$)$ ). $R^{2}$ (the coefficient of multiple determination, which indicates the extent to which estimated and actual values are linearly related[10]) is reported here for information. but is not used to assess the predictive ability of any equation.

Campbell $\epsilon t$ al. $[t]$ consider an equation acceptable if $\overline{M R E} \leq .30$ and $\operatorname{Pred}(.30) \geq .70$. Although this criterion is arbitrary, it has been widely accepted in software engineering. It is used here.

A form of trial and error was used to derive the estimation equations. Ordinary regression was used to provide initial guesses for the coefficients. A range of values around the initial guess was then defined for each coefficient, based on a visual inspection of a graph of size against the dependent variable (Drff or Cref). An exhaustive search through all combinations was then made to find the best-performed equation in terms of $\overline{M R E}$ and $\operatorname{Pred}(.30)$. This approach usually improved $\overline{M R E}$ and $\operatorname{Pred}(.30)$ substantially, compared to the regression-derived equation. 
Table 2: Best equations after high level design

\begin{tabular}{lcccc}
\hline Class & Equation & $R^{2}$ & $\overline{M R E}$ & Pred(.30) \\
\hline All programs & $6.50 \times$ Dref +150 & 0.90 & 0.31 & 0.64 \\
\hline Interactive & $5.20 \times$ Dref +510 & 0.85 & 0.22 & 0.84 \\
Computation & $5.60 \times$ Dref +170 & 0.97 & 0.23 & 0.71 \\
Translation & $6.50 \times$ Dref +150 & 0.80 & 0.36 & 0.57 \\
System & $6.50 \times$ Dref +150 & 0.91 & 0.35 & 0.60 \\
\hline filter & $7.25 \times$ Dref +110 & 0.98 & 0.34 & 0.67 \\
compression & $7.50 \times$ Dref +450 & 0.90 & 0.16 & 0.86 \\
parser & $8.87 \times$ Dref +180 & 0.93 & 0.35 & 0.67 \\
translator & $3.50 \times$ Dref +440 & 0.95 & 0.28 & 0.80 \\
\hline
\end{tabular}

Table 3: Overall accuracy after high level design

\begin{tabular}{lccc}
\hline Classes & $R^{2}$ & $\overline{M R E}$ & $\operatorname{Pred}(.30)$ \\
\hline All together & 0.90 & 0.31 & 0.64 \\
4 classes & 0.90 & 0.29 & 0.64 \\
7 classes & 0.90 & 0.27 & 0.72 \\
\hline
\end{tabular}

\section{RESULTS}

\subsection{High level design}

Table 2 shows the performance of the best equation for estimating the size of programs in each class, based on Dref. The first line gives the best equation, and its accuracy, when all programs are considered as a single class. The next four lines give this information for each broad class, and the last four lines give it for the subclasses of the file translation programs.

Two of the four broad classes (interactive and computation-intensive programs) appear to represent distinct subclasses. They were already estimated with acceptable accuracy; defining their own estimation equations improves the accuracy further.

The translation programs and system programs cannot be distinguished as useful subclasses from the original full sample. These programs were estimated most poorly in the first place. Classifying them as translation-oriented or system-oriented has gained nothing. A more finegrained classification by application is the next step to try. System programs cannot be broken down further by application. Translation programs can be broken down by application into four subclasses.

The last four lines of Table 2 show the best equations for each separate application within the translation programs. They appear to represent quite distinct subclasses. Each is estimated more accurately by its own separate equation than by the equation for the class as a whole. It appears that two subclasses are estimated with acceptable accuracy; the other two fall slightly short. With only $7-10$ programs for each application, though. firm conclusions cannot be drawn.

Overall estimation accuracy for the sample of 114 programs is shown in Table 3. The first line shows the overall accuracy when each program is estimated with the same equation. The second line shows the overall accuracy when the programs are grouped into four classes. The 
Table 4: Best equations after detailed design

\begin{tabular}{llccc}
\hline Class & \multicolumn{1}{c}{ Equation } & $R^{2}$ & $\overline{M R E}$ & $\operatorname{Pred}(.30)$ \\
\hline All programs & $4.00 \times$ Cref +30 & 0.94 & 0.24 & 0.73 \\
\hline Interactive & $3.53 \times$ Cref +300 & 0.91 & 0.18 & 0.88 \\
Computation & $3.09 \times$ Cref & 0.98 & 0.22 & 0.83 \\
Translation & $4.62 \times$ Cref & 0.85 & 0.26 & 0.69 \\
System & $4.30 \times$ Cref & 0.92 & 0.23 & 0.87 \\
\hline filter & $4.34 \times$ Cref & 0.99 & 0.23 & 0.67 \\
compression & $4.20 \times$ Cref +330 & 0.90 & 0.14 & 0.86 \\
parser & $4.55 \times$ Cref & 0.88 & 0.20 & 0.89 \\
translator & $2.40 \times$ Cref +300 & 0.94 & 0.24 & 0.80 \\
\hline
\end{tabular}

Table 5: Overall accuracy after detailed design

\begin{tabular}{lccc}
\hline Classes & $R^{2}$ & $\overline{M R E}$ & $\operatorname{Prcd}(.30)$ \\
\hline All together & 0.94 & 0.24 & 0.73 \\
4 classes & 0.93 & 0.23 & 0.81 \\
7 classes & 0.94 & 0.21 & 0.84 \\
\hline
\end{tabular}

last line shows the overall accuracy when seven classes (three broad classes, and a breakdown of the fourth into four separate applications) are used. Classifying the programs into seven classes improves $\overline{M R E}$ by $4 \%$ and $\operatorname{Pred}(.30)$ by $8 \%$. These represent relative improvements of $13 \%$ in the values of each of $\overline{M R E}$ and $\operatorname{Pred}(.30)$.

In summary, classifying programs by application has identified some distinct classes which are worth estimating separately, and overall accuracy now appears acceptable.

\subsection{Detailed design}

Table 4 shows the performance of the best equation for estimating the size of programs in each class, based on Cref. It has the same structure as Table 2.

Each class is estimated with greater accuracy by its own estimation equation. There are substantial differences between the four equations. and between each of them and the single equation used for the sample as a whole. Classifying programs broadly by application finds real differences after detailed design.

The size of interactive, computation-intensive, and system programs is estimated well as a. function of Cref. For these three classes, $\overline{M R E}$ is at most $23 \%$ and $\operatorname{Pred}(.30)$ is at least $83 \%$. Even the translation programs are almost estimated acceptably as a single class. The previous section showed that they should be broken down further by application. The last four lines of Table $t$ show the best equations when this is done. Although the accuracy generally looks good. firm conclusions cannot be drawn because the subclasses contain too few members.

Overall estimation accuracy for the sample of 114 programs is shown in Table 5 . Even without classifying the programs at all, estimation accuracy is acceptable. Classifying the programs into four classes helps; classifying them into the more sensible seven classes helps further. 


\section{CONCLUSIONS}

The main findings of this study are:

- Classifying programs by application, and using a separate estimation equation for each class, improves the recuracy of the estimates.

- When programs are classified into only a few classes, $\overline{M R E}$ and $\operatorname{Prcd}(.30)$ each improve in relative terms by about $10-1 \%$.

- For three of the four broad classes, size estimation is acceptably accurate after high level design. The fourth class needs to be broken down to a finer level, whereupon it too can be estimated with acceptable accuracy after high level design.

- It is enough to classify programs broadly by application, in order for estimates based on weighted counts of distinct variables (using equations like those in Tables 2 and 4 ) to be acceptable from high level design ouwards.

Several avenues are open for further research. A direct follow-on to this study would be to assess the value of a fine-grained classification by application. This general approach to size estimation could also be investigated with other languages. and with large industrial programs.

\section{REFERENCES}

1. Albrecht A., 'Measuring application development productivity', Proc. IBM Applications Development Symposium, October 1979, pp 83-92

2. DeMarco T., 'Controlling Software Projects', (Yourdon Press, 1982)

3. Dunsmore H., Wang A., 'A step toward early size estimation for use in productivity models', Proc. 1985 National Joint Computer Conference on Software Quality. Williamsburg VA, March 1985

4. Campbell R., Conte S.D., Rathi M.K.. 'Early prediction of software size and effort', Purdue University Technical Report SERC-TR-10-P. March 1988

5. Rathi M.K.. 'An Objective Methodology for Early Software Size Estimation'. PhD thesis. Purdue University, August 1988

6. Bourque P., C'ôté V.. 'An experiment in software sizing with structured analysis metrics'. Journal of Systems and Softuare, 1991, Vol. 15, (2), pp 159-172

¡. Verner J., Tate G.. 'A software size model', IEEE Transactions on Softuare Engineering. 1992. Vol. 18. (4), pp 265-278

8. Lokan C.J.. Conte S.D., 'Size estimation for Pascal programs', Purdue liniversity Technical Report SERC-TR-129-P. December 1992. To appear in Journal of Computer and Software Enginee ring

9. Lokan C..J.. 'Early size prediction for C' programs'. Purdue University Technical Report SERC-TR-139-P, June 1993

10. Conte S.D., Dunsmore H.. Shen V., 'Software Engineering Metrics and Models', (Benjamin('ummings, 1986) 